\title{
Directional Symmetry of the Time Lag for Downstream Absorptive Permeation studied by the Matrix Method
}

\author{
Jenn Shing Chen \\ Department of Applied Chemistry, National Chiao Tung University, Hsinchu, Taiwan, 30050, Republic \\ of China
}

\begin{abstract}
The directional symmetry of the downstream time lag for absorptive permeation, accompanying a first-order reaction, across a heterogeneous membrane has been proven by the matrix method based on the theory of Siegel. Owing to the heterogeneity the partition coefficient $(K)$, diffusivity $(D)$ and rate constant $(K)$ are all dependent on position. The directional symmetry was first shown for multiple laminates with each laminate having a distinct $K, D$ and $k$. The transmission matrix of the system is then a sequential product of those of the individual laminates. The proof is for the unit value of the determinant of the transmission matrix for each laminate and, hence, for the whole system. Directional symmetry is thus proven for the heterogeneous membrane, since it can be visualized to be an assembly of an infinite number of infinitely thin, homogeneous laminates.
\end{abstract}

A typical experiment for permeation across membranes involves maintaining, at upstream and downstream faces, the penetrant activity at constant levels $a_{\mathrm{u}}$ and $a_{\mathrm{d}}$, usually with $a_{\mathrm{u}}>a_{\mathrm{d}}=0$, respectively. The penetrant activity in the membrane is initially adjusted to $a_{\mathrm{d}}$ for running absorptive permeation or to $a_{\mathrm{u}}$ for running desorptive permeation. ${ }^{1.2}$ The accumulated amount of penetrant release is then measured as a function of time at the downstream or upstream faces. A plot of total release vs. time gives the steady-state permeability from the slope and the time lag from the intercept with the time axis. Thus four time lags are given from various combinations of upstream/downstream and desorptive/ absorptive permeation. ${ }^{1,2}$ In addition, the four time lags are also associated with reverse permeation resulting from the exchange of the upstream and downstream compartments. ${ }^{1,2}$ In conjunction with the permeability, appropriate time lags are used to determine the diffusivity and solubility (or partition coefficient) of the penetrant inside the membrane if diffusion is Fickian. ${ }^{2-4}$ They are also employed to identify and study the non-Fickian time-lag increments due to the time and/or position dependence of diffusion parameters ${ }^{5,6}$ and a consistency check of the experimental determination of various time lags. ${ }^{3,4,7}$ In practical applications, however, the diffusion time lag can be visualized as a gauge of the stability of colloid flocculation, ${ }^{8}$ or as a measure of the induction time of crystallization. ${ }^{9.10}$ These are possible because the abovementioned processes can all be modelled as diffusion under the influence of a potential field.

Consider under what conditions the downstream absorptive time lags for forward and reverse permeation are equal, i.e. the directional symmetry holds. Jaeger ${ }^{11}$ was probably the first author to address this point in the context of heat conduction across a multi-laminate slab. The extensive theoretical studies of Petropoulos et al. ${ }^{1,5,12,13}$ have revealed that the time-lag symmetry holds for any type of heterogeneity if $D$ and $K$ are functions of position, $x$, only. In the more general case where $D$ and $K$ are functions of both $x$ and concentration, $\rho$, the directional symmetry applies only if there is symmetry about the midplane of the membrane. For $K(\rho, x)$ and $D(\rho, x)$ without symmetry about the midplane, a distinction between the two cases of separable and non-separable $\rho, x$, can be made by checking the symmetry or asymmetry of flux, respectively. Those results are summarized in Table IV of ref. 5 , and serve a practical, diagnostic purpose. Up to now, however, only permeation across heterogeneous membranes in the absence of chemical reactions has been considered.

Of increasing practical importance are membranes with associated reactive moieties, such as catalysts or enzymes, to enhance the productivity of chemical and biological processes in catalytic membrane reactors, ${ }^{14}$ to enhance the performance of biosensors, ${ }^{15}$ or to simulate active transport using uneven distribution of enzyme activities. ${ }^{16}$ A theoretical study ${ }^{17}$ has also suggested that the directionality of products and substrate fluxes and the separation of product and substrate can be drastically improved by using an appropriate, non-uniform distribution of the reactive moieties. Hence, it is desirable to extend the discussion of directional symmetry to include the case where the reaction takes place inside the membrane. Only first-order reactions with positiondependent rate constants are considered here. This simplification will, of course, not cover all cases, especially for the enzyme catalytic reaction which usually has a MichaelisMenten type rate constant. However, as pointed out by Kubin and Spacek, ${ }^{16}$ in the case of a linear gradient distribution of the enzyme with high or low concentrations of substrate, the accompanying first-order reaction will have a position-dependent rate constant.

The matrix method will be employed to prove the directional symmetry of the downstream time lag for absorptive permeation across a heterogeneous membrane accompanying a first-order reaction. In a previous publication ${ }^{18}$ the directional symmetry of the time lag for the same system was proven using the symmetry property with respect to the exchange of two coordinate arguments of the Green's function associated with the diffusion equation.

A review of the matrix formulation of the mass diffusion (or heat conduction) problem can be found in ref. 19-21.

\section{Transmission Matrix of a Homogeneous Membrane}

Consider one-dimensional absorptive permeation across a homogeneous membrane extending from $x=x_{\mathrm{u}}$ to $x=x_{\mathrm{d}}$, with $x_{\mathrm{d}}>x_{\mathrm{u}}$. The penetrant is also involved in a first-order reaction with rate constant $k$ inside the membrane. The concentrations of penetrant in the upstream compartment (at the left side of the membrane) and downstream compartment (at the right side of the membrane), $\rho_{\mathrm{u}}(t)$ and $\rho_{\mathrm{d}}(t)$, respectively, are time-dependent. The permeation experiment starts with zero concentration of penetrant inside the membrane. The 
mathematical formulation is then

$$
\begin{aligned}
\frac{\partial}{\partial t} \rho(x, t)=D & \frac{\partial^{2}}{\partial x^{2}} \rho(x, t)-k \rho(x, t) ; \\
& \rho(x, 0)=0, \quad \frac{\rho\left(x_{\mathrm{u}}, t\right)}{K}=\frac{\rho_{\mathrm{u}}(t)}{K_{\mathrm{u}}}, \frac{\rho\left(x_{\mathrm{d}}, t\right)}{K}=\frac{\rho_{\mathrm{d}}(t)}{K_{\mathrm{d}}}
\end{aligned}
$$

where $D$ is the diffusivity of the penetrant in the membrane; $\rho(x, t), \rho\left(x_{\mathrm{u}}, t\right), \rho\left(x_{\mathrm{d}}, t\right)$ represent, respectively, the concentration inside the membrane and at the upstream and downstream sides. $K, K_{\mathrm{u}}, K_{d}$ are partition coefficients in the membrane, upstream and downstream compartments. The boundary conditions take into account the requirement that the activity of the penetrant $(=\rho / K)$ should be continuous at interfaces.

We will analyse the problem in the Laplace domain Expressing eqn. (1) in terms of $s$, the Laplace variable, and $\hat{\rho}(x, s)$, the Laplace transform of $\rho(x, t)$, gives

$$
\begin{aligned}
& s \hat{\rho}(x, s)-\rho(x, 0)=D \frac{\mathrm{d}^{2}}{\mathrm{~d} x^{2}} \hat{\rho}(x, s)-k \hat{\rho}(x, s) ; \\
& \qquad \frac{\hat{\rho}\left(x_{\mathrm{u}}, s\right)}{K}=\frac{\hat{\rho}_{\mathrm{u}}(s)}{K_{\mathrm{u}}}, \frac{\hat{\rho}\left(x_{\mathrm{d}}, s\right)}{K}=\frac{\hat{\rho}_{\mathrm{d}}(s)}{K_{\mathrm{d}}}
\end{aligned}
$$

With $\rho(x, 0)=0$, the solution to eqn. (2) is given by

$$
\begin{aligned}
\hat{\rho}(x, s)= & \frac{K}{\sinh \left[q\left(x_{\mathrm{d}}-x_{\mathrm{u}}\right)\right]}\left\{\frac{\hat{\rho}_{\mathrm{u}}(s)}{K_{\mathrm{u}}} \sinh \left[q\left(x_{\mathrm{d}}-x\right)\right]\right. \\
& \left.+\frac{\hat{\rho}_{\mathrm{d}}(s)}{K_{\mathrm{d}}} \sinh \left[q\left(x-x_{\mathrm{u}}\right)\right]\right\}
\end{aligned}
$$

with $q=(s+k / D)^{1 / 2}$. The permeation fluxes at the upstream side $\left(x=x_{\mathbf{u}}\right)$ and downstream side $\left(x=x_{\mathrm{d}}\right)$ are then

$$
\begin{aligned}
& \hat{J}_{\mathrm{u}}(s)=D q K\left\{\frac{\hat{\rho}_{\mathrm{u}}(s)}{K_{\mathrm{u}}} \operatorname{coth}(q h)-\frac{\hat{\rho}_{\mathrm{d}}(s)}{K_{\mathrm{d}}} \operatorname{csch}(q h)\right\} \\
& \hat{J}_{\mathrm{d}}(s)=D q K\left\{\frac{\hat{\rho}_{\mathrm{u}}(s)}{K_{\mathrm{u}}} \operatorname{csch}(q h)-\frac{\hat{\rho}_{\mathrm{d}}(s)}{K_{\mathrm{d}}} \operatorname{coth}(q h)\right\}
\end{aligned}
$$

where $h\left(=x_{\mathrm{d}}-x_{\mathrm{u}}\right)$ is the thickness of the membrane, and the positive direction of the fluxes is defined to be from left to right. Eqn. (4) and (5) can be rewritten in a matrix form

$$
\left[\begin{array}{c}
\frac{\hat{\rho}_{\mathrm{d}}(s)}{K_{\mathrm{d}}} \\
\hat{J}_{\mathrm{d}}(s)
\end{array}\right]=\left[\begin{array}{cc}
\cosh (q h) & -\frac{\sinh (q h)}{D q K} \\
-D q K \sinh (q h) & \cosh (q h)
\end{array}\right]\left[\begin{array}{c}
\frac{\hat{\rho}_{\mathrm{u}}(s)}{K_{\mathrm{u}}} \\
\hat{J}_{\mathrm{u}}(s)
\end{array}\right]
$$

The $2 \times 2$ array in eqn. (6) is the transmission matrix for absorptive permeation across a homogeneous membrane.

\section{Directional Symmetry of Downstream Time Lags for Absorptive Permeation accompanying Chemical Reactions across Multiple-laminate and/or Heterogeneous Membranes}

Consider a membrane which consists of a series of $n$ different homogeneous laminates and extends from $x=x_{u}$ to $x=x_{\mathrm{d}}$. A typical $i$ th slab $(i=1,2, \ldots, n)$, extending from $x=x_{i-1}$ to $x=x_{i}$, is characterized by a thickness $d_{i}$, a diffusitivity $D_{i}$, a partition coefficient $K_{i}$ and a first-order rate constant $k_{i}$. Thus we have $d_{i}=x_{i}-x_{i-1}, x_{0}=x_{u}$ and $x_{n}=x_{d}$. The initial and boundary conditions are the same as in eqn. (1). In terms of transmission matrices the permeation problem is formulated as

$$
\left[\begin{array}{c}
\frac{\hat{\rho}_{\mathrm{d}}(s)}{K_{\mathrm{d}}} \\
\hat{J}_{\mathrm{d}}(s)
\end{array}\right]=T_{n}(s) T_{n-1}(s), \ldots, T_{1}(s)\left[\begin{array}{c}
\frac{\hat{\rho}_{\mathrm{u}}(s)}{K_{\mathrm{u}}} \\
\hat{J}_{\mathrm{u}}(s)
\end{array}\right]=T(s)\left[\begin{array}{c}
\frac{\hat{\rho}_{\mathrm{u}}(s)}{K_{\mathrm{u}}} \\
\hat{J}_{\mathrm{u}}(s)
\end{array}\right]
$$

where $T(s)$ is the resultant transmission matrix of the whole membrane. $T_{i}(s)$, the transmission matrix of the $i$ th component laminate, is in the same form as the transmission matrix in eqn. (6) except for $D, K, q$ and $h$ being attached by a subscript $i$.

The transport equation, eqn. (7), can be converted to

$$
\left[\begin{array}{c}
\hat{\rho}_{\mathrm{u}}(s) \\
K_{\mathrm{u}} \\
\hat{J}_{\mathrm{u}}
\end{array}\right]=T^{-1}(s)\left[\begin{array}{c}
\frac{\hat{\rho}_{\mathrm{d}}(s)}{K_{\mathrm{d}}} \\
\hat{J}_{\mathrm{d}}(s)
\end{array}\right]
$$

This is possible because the determinant of every component matrix $T_{i}(x)(i=1, \ldots, n)$ is unity, and hence the determinant of $T(s)$ is also unity, i.e. $T(s)$ is non-singular. Let the $2 \times 2$ matrix, $T(s)$, be represented by a general form as

$$
T(s)=\left[\begin{array}{ll}
\alpha(s) & \beta(s) \\
\gamma(s) & \delta(s)
\end{array}\right]
$$

where $\alpha(s), \beta(s), \gamma(s), \delta(s)$ are real numbers. With unit determinant of $T(s)$, its inverse becomes

$$
T^{-1}(s)=\left[\begin{array}{rr}
\delta(s) & -\beta(s) \\
-\gamma(s) & \alpha(s)
\end{array}\right]
$$

As mentioned before, exchanging upstream and downstream compartments results in flow reversal. It is natural to define, in reverse permeation, the positive direction to be from right to left. Schematically

$$
\left[\begin{array}{c}
\frac{\hat{\rho}_{\mathrm{u}}(s)}{K_{\mathrm{u}}} \\
-\hat{J}_{\mathrm{u}}(s)
\end{array}\right]^{\mathrm{R}}=\left[\begin{array}{c}
\hat{\rho}_{\mathrm{d}}(s) \\
K_{\mathrm{d}} \\
\hat{J}_{\mathrm{d}}(s)
\end{array}\right] ;\left[\begin{array}{c}
\frac{\hat{\rho}_{\mathrm{d}}(s)}{K_{\mathrm{d}}} \\
-\hat{J}_{\mathrm{d}}(s)
\end{array}\right]^{\mathrm{R}}=\left[\begin{array}{c}
\frac{\hat{\rho}_{\mathrm{u}}(s)}{K_{\mathrm{u}}} \\
\hat{J}_{\mathrm{u}}(s)
\end{array}\right]
$$

where the superscript $R$ is used to signify the reverse permeation. A combination of eqn. (8), (10) and (11) yields

$$
\left[\begin{array}{c}
\frac{\hat{\rho}_{\mathrm{d}}(s)}{K_{\mathrm{d}}} \\
\hat{J}_{\mathrm{d}}
\end{array}\right]^{\mathrm{R}}=\left[\begin{array}{ll}
\delta(s) & \beta(s) \\
\gamma(s) & \alpha(s)
\end{array}\right]\left[\begin{array}{c}
\frac{\hat{\rho}_{\mathrm{u}}(s)}{K_{\mathrm{u}}} \\
\hat{J}_{\mathrm{u}}(s)
\end{array}\right]^{\mathrm{R}}
$$

This is the transport equation of reverse absorptive permeation formulated in matrix form.

According to Siegel ${ }^{21}$ the downstream absorptive time lag can be calculated from the transmission matrix element as

$$
L=\lim _{s \rightarrow 0} \frac{\mathrm{d} T_{12}(s) / \mathrm{d} s}{T_{12}(s)}
$$

where $T_{12}(s)$ is the element of first row and second column of $T(s)$. For both forward and reverse permeation $T_{12}(s)$ is identified to be $\beta(s)$. Thus it is readily seen that the downstream absorptive time lags for forward permeation, $L$, and those for reverse permeation, $L^{\mathrm{R}}$, are identical, i.e.

$$
L=L^{\mathbf{R}}=\lim _{s \rightarrow 0} \frac{\mathrm{d} \beta(s) / \mathrm{d} s}{\beta(s)}
$$

A heterogeneous membrane, in which $D, K$ and $k$ are continuously varying with position, can be visualized as an assembly of $n$ infinitely thin, homogeneous laminates with $n \rightarrow \infty$, keeping the total thickness equal to the original one Since the preceding proof is valid irrespective of the value of $n$, and the thickness of each laminate, the directional symmetry obviously holds for absorptive permeation accompanying a first-order reaction across a heterogeneous membrane.

The work was funded in part by the National Science Council, Taiwan, Republic of China under the project NSC 82-0208-M-009-019.

\section{References}

1 J. H. Petropoulos and P. P. Roussis, J. Chem. Phys., 1967, 47, 1491. 
2 J. H. Petropoulos, Adv. Polym. Sci., 1985, 64, 93.

3 K. Tsimillis and J. H. Petropoulos, J. Phys. Chem., 1977, 81, 2185.

4 C. Savvakis and J. H. Petropoulos, J. Phys. Chem., 1982, 86, 5128.

5 J. H. Petropoulos and P. P. Roussis, J. Chem. Phys., 1969, 50, 3951.

6 J. H. Petropoulos and P. P. Roussis, J. Chem. Phys., 1969, 51, 1332.

7 P. P. Roussis and J. H. Petropoulos, J. Chem. Soc., Faraday Trans. 2, 1976, 72, 737.

8 R. D. Vold and M. J. Vold, Colloid and Interface Chemistry, Addison-Wesley, London, 1983

9 H. L. Frisch, J. Chem. Phys., 1957, 27, 90.

10 H. L. Frisch and C. C. Carter, J. Chem. Phys., 1971, 54, 4326.

11 J. C. Jaeger, Quart. Appl. Math., 1950, 8, 187.
12 J. H. Petropoulos, P. P. Roussis and J. Petrou, J. Colloid Interface Sci., 1977, 62, 114.

13 J. H. Petropoulos and P. P. Roussis, J. Chem. Phys., 1967, 47, 1496.

14 T. Naka and N. Sakamoto, J. Membrane Sci., 1992, 74, 159.

15 W. R. Vieth, Membrane Systems: Analysis and Design, Hanser Publishers, Munich, 1988.

16 M. Kubin and P. Spacek, Polymer, 1973, 14, 505

17 T. Ciftci and W. R. Vieth, J. Mol. Catal., 1980, 8, 455.

18 J. S. Chen and F. Rosenberger, Chem. Eng. Commun., 1991, 104, 41.

19 A. H. van Gorcum, Appl. Sci. Res. A, 1951, 2, 272.

20 B. Bunow and R. Aris, Math. Biosci., 1975, 26, 157

21 R. A. Siegel, J. Phys. Chem., 1991, 95, 2556.

Paper 3/06714I; Received 9th November, 1993 